\title{
Die Kosten der Depression und die Wirtschaftlichkeit ihrer Behandlung
}

\author{
The Costs of Depression and the Cost-Effectiveness of Treatment
}

\author{
Autoren \\ Hans-Helmut König ${ }^{1}$, Melanie Luppa ${ }^{2}$, Steffi Riedel-Heller ${ }^{3}$ \\ Institute \\ Universitätsklinikum Hamburg-Eppendorf, Institut für Medizinische Soziologie, Sozialmedizin und Gesundheitsökonomie \\ 2 Public Mental Health Research Unit, Zentrum für Psychische Gesundheit, Klinik und Poliklinik für Psychiatrie, \\ Universität Leipzig \\ Selbstständige Abteilung für Sozialmedizin \& Professur für Public Health, Klinik und Poliklinik für Psychiatrie \\ und Psychotherapie, Universität Leipzig
}

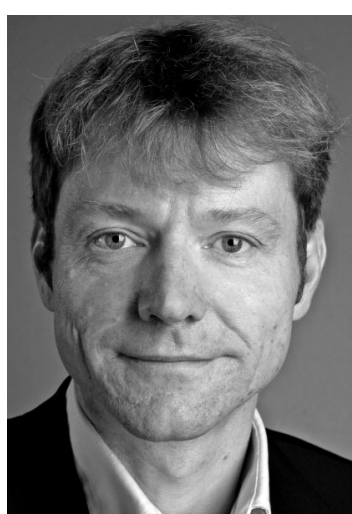

Prof. Hans-Helmut König
Bibliografie

DOI http://dx.doi.org/

$10.1055 / \mathrm{s}-0030-1248510$

Psychiat Prax 2010;

37: 213-215

(c) Georg Thieme Verlag KG

Stuttgart · New York.

ISSN 0303-4259

Korrespondenzadresse

Prof. Dr. Hans-Helmut König,

M.P.H.

Universitätsklinikum HamburgEppendorf

Institut für Medizinische

Soziologie, Sozialmedizin und

Gesundheitsökonomie

Martinistraße 52

20246 Hamburg

h.koenig@uke.uni-hamburg.de

\section{Depressionen häufig und folgenschwer \\ $\nabla$}

Depressive Erkrankungen sind häufig und folgenschwer. Die 12-Monats-Prävalenz depressiver Störungen bei über 18-jährigen Personen in der deutschen Allgemeinbevölkerung beträgt nach den Ergebnissen des Bundesgesundheitssurveys 10,7\% [1]. Das bedeutet, in Deutschland sind ca. 5-6 Millionen erwachsene Bundesbürger pro Jahr von einer Depression betroffen. Es wird davon ausgegangen, dass Depressionen bis zum Jahr 2020 neben den koronaren Herzerkrankungen weltweit die führende Ursache für vorzeitigen Tod und durch Behinderung beeinträchtigte Lebensjahre sein werden [2-4]. Depressive Erkrankungen führen zu einer verringerten Leistungsfähigkeit [5], einem Verlust an gesundheitsbezogener Lebensqualität [6] und einer erhöhten Mortalität [7], vor allem aufgrund von Suiziden. So versterben $15 \%$ der Patienten mit schweren depressiven Erkrankungen durch Suizid [8] und ca. $60 \%$ aller Suizide sind auf Depressionen zurückzuführen. Menschen mit depressiven Erkrankungen gehören zu den Vielnutzern des Gesundheitssystems. Deshalb interessieren sich Gesundheitsökonomen schon seit einigen Jahren für diese Krankheitsgruppe [9]. Das vorliegende Editorial fragt nach dem Stand der Forschung zu den Kosten depressiver Erkrankungen und der Wirtschaftlichkeit ihrer Behandlung.

\section{Krankheitskostenstudien und methodische Ansätze \\ $\nabla$}

Krankheitskostenstudien zielen auf die Beschreibung der Inanspruchnahme von medizinischen Leistungen und die Berechnung der krankheitsbedingten Kosten. Die Gesamtkosten einer Erkrankung setzen sich dabei zum einen aus den direkten Kosten oder den Versorgungskosten und zum anderen aus den indirekten Kosten oder den
Produktionsausfallkosten zusammen. Zu den direkten Kosten zählen medizinische und nicht medizinische Versorgungskosten. Indirekte Kosten kommen durch Morbiditätskosten (Arbeitsunfähigkeit, verminderte Produktivität am Arbeitsplatz bzw. verminderte Erwerbsfähigkeit) als auch durch Mortalitätskosten (verlorene Lebenszeit) zustande. Für die Ermittlung von Krankheitskosten gibt es verschiedene methodische Ansätze. Zum einen unterscheiden wir den Prävalenz- vom Inzidenzansatz. Im ersten Fall werden die Kosten prävalenter Fälle in einer Zeitperiode berechnet, im zweiten werden die Kosten inzidenter Fälle über die Restlebenszeit ermittelt. Zum anderen wird ein Top-down-Ansatz von einem Bottom-up-Ansatz unterschieden. Im Topdown-Ansatz werden die Krankheitskosten auf der Grundlage der Desaggregation von Sekundärdaten berechnet. Im Rahmen des Bottom-up-Ansatzes erfolgt die Kostenmessung direkt beim einzelnen Patienten mittels Fragebögen zur Leistungsinanspruchnahme, aus Patientenakten oder Abrechnungsdaten der Kostenträger. Des Weiteren unterscheiden wir einen indikationsspezifischen Kostenmessansatz von einem sog. Inkrementalkostenansatz. Beim indikationsspezifischen Kostenmessansatz werden ausschließlich indikationsspezifische Versorgungsleistungen und Arbeitsausfallzeiten berücksichtigt. Der Inkrementalkostenansatz zielt auf die Berechnung der Differenz in den Gesamtkosten zwischen Erkrankten und gesunden Kontrollpersonen als sog. excess costs.

\section{Direkte Kosten der Depression $\nabla$}

Zahlen des Statistischen Bundesamtes für die direkten Krankheitskosten in Deutschland 2006 nach Krankheitsgruppen weisen auf der Grundlage prävalenzbasierter indikationsspezifischer Kostenmessung mit dem Top-down-Ansatz Kos- 
ten von 26,7 Milliarden Euro für psychische Erkrankungen und Verhaltensstörungen aus [10]. Diese machen $11 \%$ der Gesamtkosten aus. Auf die affektiven Störungen entfallen hier 5,1 Milliarden Euro, fast 1 / 5 aller direkten Kosten für psychische Erkrankungen. Der Großteil entfällt hier mit 4,6 Milliarden Euro auf depressive Erkrankungen.

Wie stellen sich die Kosten depressiver Erkrankungen in der internationalen Literatur dar? Melanie Luppa et al. [11] legten im Journal of Affective Disorders eine systematische Übersichtsarbeit vor, die auf einer umfassenden Literatursuche in Medline, Web of Science, der Cochrane Library und des PSYCHINDEXplus mit ca. 4300 Treffern basiert. Letztlich kamen 24 Krankheitskostenstudien zur Auswertung. Die Ergebnisse beziehen sich auf prävalenzbasierte Bottom-up-Studien. Studien mit indikationsspezifischer Kostenmessung (also ohne Kontrollgruppe) konnten zeigen, dass die durchschnittlichen direkten Kosten je Patient und Jahr 1345-2746 USD-Kaufkraftparitäten (USD-KKP) in Preisen von 2003 betragen. Studien mit Inkrementalkostenansatz zeigten, dass Depressive im Vergleich zu Nichtdepressiven bis zu 5-fach erhöhte direkte Kosten haben. Besonders herauszustellen ist, dass sehr hohe Kosten außerhalb der psychiatrischen Versorgung auftraten. Zusammenfassend kann gesagt werden, dass in den meisten Studien die direkten Kosten je Patient und Jahr zwischen 1000-2500 USD-KKP lagen. Auf der Grundlage der European Study of the Epidemiology of Mental Disorders (ESEMeD) berichteten Friemel et al. $[12,13]$ die direkten Kosten von depressiven Erkrankungen in Deutschland auf der Grundlage einer bevölkerungsrepräsentativen Befragung (prävalenzbasierte Bottom-up-Studie mit indikationsspezifischer Kostenmessung). Bezogen auf alle Befragten mit einer 12-Monats-Prävalenz einer Depression $(n=131)$ konnten durchschnittliche Kosten von 668 Euro berechnet werden. Diejenigen Befragten mit einer Depression, die deshalb auch Leistungen in Anspruch nahmen $(n=72)$, verursachten im Mittel 1264 Euro Kosten, wobei hier der Großteil auf die stationäre Versorgung (53\%) entfiel, gefolgt von der ambulanten Behandlung mit 34\%. Lediglich 9\% wurden für Medikamente ausgegeben.

Während sich eine substanzielle Zahl von Studien mit den Kosten depressiver Erkrankungen im Erwachsenenalter beschäftigt, existieren nur wenige Studien, die sich mit den Kosten depressiver Störungen im Alter befassen [14-16]. Depressionen im Alter stellen eine außerordentlich wichtige Diagnosegruppe dar. Sie sind nach den demenziellen Erkrankungen die wichtigsten gerontopsychiatrischen Erkrankungen. Aufgrund der demografischen Entwicklung wird dieses Feld an Relevanz gewinnen. In einer Studie, die die direkten Kosten der Depression im Alter bei einer repräsentativen Stichprobe von über 75-jährigen Leipziger Allgemeinarztpatienten untersuchte (prävalenzbasierte Bottomup-Studie mit Inkrementalkostenansatz) konnten wir zeigen, dass Depressive signifikant erhöhte direkte Kosten im Vergleich zu Nichtdepressiven aufweisen, wobei die Kosten der Depressiven um durchschnittlich 1593 Euro höher als die der nicht depressiven Senioren sind. In einer Regressionsanalyse zu Einflussfaktoren auf die direkten Kosten zeigte sich, dass depressive Erkrankungen einen ganz eigenständigen signifikanten Beitrag leisten und sich die direkten Kosten mit jedem zusätzlichen Punkt auf der geriatrischen Depressionsskala um 336 Euro erhöhen [17]. In diesem Sample von Senioren konnten wir auch die direkten Kosten von Menschen mit erkannter und nicht erkannter Depression im Alter vergleichen [18]. Die höchsten Kosten hatten diejenigen Senioren, deren Depression vom Hausarzt unerkannt war (5582 Euro), gefolgt von den Personen, deren De- pression erkannt war (4722 Euro). Die geringsten Kosten hatten nicht depressive Senioren mit durchschnittlich 3648 Euro [18].

\section{Indirekte Kosten}

$\nabla$

Depressive Erkrankungen haben hohe indirekte Kosten. Das Statistische Bundesamt berichtet verlorene Erwerbstätigkeitsjahre durch Morbidität und Mortalität in Deutschland im Jahr 2006. Dabei entfällt auf die psychischen Erkrankungen und Verhaltensstörungen mit 638000 verlorenen Erwerbstätigkeitsjahren ein substanzieller Teil (16\%) aller verlorenen Erwerbstätigkeitsjahre. Ein Viertel davon entfallen auf depressive Erkrankungen. In der internationalen Literatur werden auf der Grundlage von prävalenzbasierten Top-down-Studien durchschnittliche Morbiditätskosten von 2000-3700 USD-KKP pro Jahr berichtet, die Mortalitätskosten belaufen sich durchschnittlich auf 200-400 USD-KKP [11]. Damit sind die indirekten Kosten deutlich höher als die direkten Kosten. Oft werden indirekte Kosten eher unterschätzt, da selten unbezahlte Arbeit berücksichtigt wird. Zudem ist häufig eine schlechtere Ausbildung und eine geringere Produktivität am Arbeitsplatz (Presenteismus) die Folge von Depressionen, was die indirekten Kosten weiter erhöht.

\section{„Rechnet“ sich die Depressionsbehandlung? \\ $\nabla$}

Depressive Störungen sind eine häufige Krankheitsgruppe, die mit erheblichen Kosten verbunden ist. Auch wenn man hierzulande eine offene Diskussion aus ethischen Gründen eher scheut, erhebt sich aus Sicht der Kostenträger doch die Frage, ob durch eine optimierte Behandlung die direkten medizinischen Versorgungskosten reduziert werden könnten. Dabei ist zu beachten, dass die depressionsspezifischen Behandlungskosten nur einen kleinen Teil der gesamten Versorgungskosten von depressiven Patienten ausmachen. Die zusätzlichen Kosten einer optimierten Behandlung könnten durch Einsparungen bei anderen Versorgungsleistungen ausgeglichen werden. In der angelsächsischen Literatur wird dies schon länger diskutiert. Die Studienergebnisse dazu, die in Übersichtsarbeiten von Donohue et al. [19] und Wang et al. [20] zusammengefasst werden, sind uneinheitlich. Werden also durch eine optimierte Behandlung die direkten medizinischen Versorgungskosten reduziert? - eher nicht. Etwas anders fällt die Antwort aus, wenn man aus der gesamtgesellschaftlichen Sicht danach fragt, ob durch eine optimierte Behandlung die Gesamtkosten der Depression reduziert werden. Dahinter steht die Frage, ob die zusätzlichen Kosten einer optimierten Behandlung durch die Reduktion der indirekten Kosten, also durch die Erhöhung der Produktivität, kompensiert werden. Dazu liegen nur wenige Studien vor. Auch hier gibt es inkonsistente Befunde, jedoch gehen die meisten Hinweise in die Richtung, dass es zu einer Reduktion der Gesamtkosten kommt $[19,20]$.

Anders gelagert ist die Frage, ob eine optimierte Behandlung der Depression kosteneffektiv ist. Stehen also die Kosten einer optimierten Behandlung in einem günstigen Verhältnis zu den gesundheitlichen Effekten? Hier fällt die Antwort eindeutig aus. Zahlreiche Studien berichten günstige inkrementelle Kosten-Effektivitäts-Relationen (IKER) für eine optimierte Behandlung der Depression [19,21]. So liegt die IKER für eine optimierte Behandlung im Rahmen der Primärversorgung häufig unter 20000 USD pro QALY [19] und damit deutlich unter dem oft zitierten Schwel- 
lenwert von 50000 USD pro QALY, bis zu dem Gesundheitsleistung im Allgemeinen als kosteneffektiv angesehen werden. Es existieren somit kosteneffektive Behandlungen der Depression, die aus gesamtgesellschaftlicher Perspektive vermutlich sogar kostensparend sind, wenn indirekte Kosten berücksichtigt werden. Hier wird noch weitere Forschungsarbeit benötigt, um dies $\mathrm{zu}$ untermauern. Auch andere Fragen sind noch weitgehend unbeantwortet. Zum Beispiel die Frage, wie hoch die indirekten Kosten durch eine reduzierte Produktivität am Arbeitsplatz (Presenteismus) sind und wie hoch die Kosten einer unerkannten und unbehandelten Depression sind. Dazu hatten wir in einer eigenen Studie erste Pilotdaten vorgelegt [17], die jedoch auf eine breitere Datenbasis gestellt werden müssen.

Ein spannendes Forschungsfeld. Obgleich für viele Behandler, die den einzelnen Menschen in seiner Lebenswelt im Blickfeld haben, die gesundheitsökonomische Perspektive gewöhnungsbedürftig ist, unterstreichen diese Ergebnisse die Relevanz psychischer Störungen, insbesondere depressiver Erkrankungen $[22,23]$.

\section{Literatur}

1 Jacobi F, Wittchen HU, Holting C et al. Prevalence, co-morbidity and correlates of mental disorders in the general population: results from the German Health Interview and Examination Survey (GHS). Psychol Med 2004; 34: 597-611

2 Murray CJL, Lopez AD. Alternative visions of the future: projecting mortality and disability, 1990-2020. In: Murray CJL, Lopez AD, Hrsg. The Global Burden of Disease. A comprehensive assessment of mortality and disability from diseases, injuries and risk factors in 1990 and projected to 2020. Publication from the Harvard School of Public Health, on behalf of the World Health Organization and the World Bank Cambridge. Cambridge: MA: Harvard University Press, 1996: 361-395

3 Richter D, Berger K, Reker T. Are mental disorders on the increase? A systematic review. Psychiat Prax 2008; 35: 321-330

4 Spiessl H, Jacobi F. Do mental disorders really increase? Psychiat Prax 2008; 35: 318-320

5 Alexopoulos GS, Vrontou C, Kakuma T et al. Disability in geriatric depression. Am J Psychiatry 1996; 153: 877-885

6 Unützer J, Patrick DL, Diehr P et al. Quality adjusted life years in older adults with depressive symptoms and chronic medical disorders. Int Psychogeriatr 2000; 12: 15-33
7 Schulz R, Drayer RA, Rollman BL. Depression as a risk factor for non-suicide mortality in the elderly. Biol Psychiatry 2002; 3: 205-225

8 Angst J, Angst F, Stassen HH. Suicide risk in patients with major depressive disorder. J Clin Psychiatry 1999; 60 (Suppl. 2): 57-62

9 König HH, Friemel S. Gesundheitsökonomie psychischer Krankheiten. Bundesgesundheitsblatt Gesundheitsforschung Gesundheitsschutz 2006; 49: 46-56

10 http://www.destatis.de/jetspeed/portal/cms/Sites/destatis/Internet/ $\mathrm{DE} /$ Content/Statistiken/Gesundheit/Krankheitskosten/Aktuell,templa teId=renderPrint.psml

11 Luppa M, Heinrich S, Angermeyer MC et al. Cost-of-illness studies of depression: a systematic review. J Affect Disord 2007; 98: 29-43

12 Friemel S, Bernert S, Angermeyer MC et al. The direct costs of depressive disorders in Germany. Psychiat Prax 2005; 32: 113-121

13 Günther $\mathrm{OH}$, Friemel S, Bernert $\mathrm{S}$ et al. The burden of depressive disorders in Germany - results from the European Study of the Epidemiology of Mental Disorders (ESEMeD). Psychiat Prax 2007; 34: 292-301

14 Katon WJ, Lin E, Russo J et al. Increased medical costs of a populationbased sample of depressed elderly patients. Arch Gen Psychiatry 2003; 60: 897-903

15 Unützer J, Patrick DL, Simon G et al. Depressive symptoms and the cost of health services in HMO patients aged 65 years and older. A 4-year prospective study. JAMA 1997; 277: 1618-1623

16 Callahan CM, Hui SL, Nienaber NA et al. Longitudinal study of depression and health services use among elderly primary care patients. J Am Geriatr Soc 1994; 42: 833-838

17 Luppa M, Heinrich S, Matschinger $H$ et al. Direct costs associated with depression in old age in Germany. J Affect Disord 2008; 105: 195-204

18 Luppa M, Heinrich S, Angermeyer MC et al. Healthcare costs associated with recognized and unrecognized depression in old age. Int Psychogeriatr 2008; 20: 1219-1229

19 Donohue JM, Pincus HA. Reducing the societal burden of depression: a review of economic costs, quality of care and effects of treatment. Pharmacoeconomics 2007; 25: 7-24

20 Wang PS, Simon G, Kessler RC. The economic burden of depression and the cost-effectiveness of treatment. Int J Methods Psychiatr Res 2003; 12: $22-33$

21 Barrett B, Byford S, Knapp M. Evidence of cost-effective treatments for depression: a systematic review. J Affect Disord 2005; 84: 1-13

22 Bramesfeld A, Riedel-Heller S. Priorities in mental health services research. Psychiat Prax 2008; 35: 315-317

23 Riedel-Heller S, Bramesfeld A, Roick C et al. Call for more health services research. Psychiat Prax 2008; 35: 157-159 